\title{
Estudio y seguimiento nutricional en una población de ancianos de un centro geriátrico
}

\author{
Laura Guzmán Díaz ${ }^{1}$, Herminia López García de la Serrana ${ }^{1}$ y María Jesús \\ Oliveras López ${ }^{2}$ \\ ${ }^{1}$ Universidad de Granada (España); ${ }^{2}$ Universidad Pablo Olavide-Sevilla (España)
}

Valorar nutricionalmente al anciano nos va a permitir conseguir y mantener un buen estado nutricional en la población anciana. Objetivos: 1. Valoración del estado nutricional de la población. 2. Evaluación de la ingesta de la población de acuerdo a las Ingestas recomendadas. 3. Valoración de la capacidad antioxidante del menú y comparación con la media española. Material y métodos: El estudio se realizó con 28 ancianos. Se utilizaron indicadores sociodemográficos, datos antropométricos, tensión arterial, cuestionarios de valoración del estado nutricional y valoración de la ingesta. Además, se determinó la capacidad antioxidante del menú por la técnica de ORAC. Resultados: Los varones presentaron mayor sobrepeso y las mujeres mayor obesidad. El índice de cintura-cadera fue mayor en hombres $(0,94 \pm 0,08 \mathrm{~cm})$. Referente a la ingesta, existe riesgo de desnutrición superior al 50\% (MNA y NSI), exceso de colesterol y déficit de ácido fólico, vitamina D y magnesio en ambos sexos. La capacidad antioxidante fue de $31.377 \mu$ moles/día y los polifenoles totales de $2.344 \mathrm{mg} /$ día. Conclusiones: Existe un elevado riesgo de desnutrición (MNA, NSI) y de padecer enfermedades cardiovasculares (ICC) entre los participantes. La capacidad antioxidante del menú triplica los valores de referencia y el perfil calórico de los ancianos es inadecuado, con exceso en grasas y colesterol y bajo en carbohidratos.

Palabras clave: Desnutrición, valoración nutricional, sobrepeso, ancianos, nutrición, capacidad antioxidante.

Study and nutritional monitoring in a population of elderly in a geriatric center. Rate the elderly nutritionally will allow us to achieve and maintain good nutritional status in the elderly population. Objectives: 1 Assessment of nutritional status of the population.. Two. Evaluation of population intake according to recommended intakes. Three. Valuations menu and antioxidant capacity compared to the Spanish average. Methods: The study was conducted with 28 elders. Sociodemographic indicators, anthropometric data, blood pressure, questionnaires assessing the nutritional status and assessment of intake were used. In addition, the antioxidant capacity of menu technique ORAC was determined. Results: Males had higher overweight and obesity increased women. The waist-hip ratio was higher in men $(0.94 \pm 0.08 \mathrm{~cm})$. Regarding the intake, there is greater risk of malnutrition $50 \%$ (MNA and NSI), excess cholesterol and folic acid deficiency, vitamin D and magnesium in both sexes. The antioxidant capacity was 31,377 umol / day and total polyphenols of 2,344 $\mathrm{mg} /$ day. Conclusions: There is a high risk of malnutrition (MNA, NSI) and cardiovascular disease (ICC) among participants. The antioxidant capacity of the menu three times the reference values and the energy profile of the elderly is inadequate, with excess fat and cholesterol and low in carbohydrates.

Keywords: Malnutrition, nutritional assessment, overweight, elderly, nutrition, antioxidant capacity.

Correspondencia: Laura Guzmán Díaz. Facultad de Farmacia. Universidad de Granada. Campus Universitario de Cartuja. C.P.: 18071. Granada (España). E-mail: laura_5851@hotmail.com 
El envejecimiento es un proceso fisiológico que acompaña a todos los seres vivos desde el momento de su nacimiento. Existen dos tipos de envejecimiento: intrínseco, primario o fisiológico, que es aquel que está condicionado por la carga genética con la que nace cada individuo y el envejecimiento extrínseco o secundario que a su vez está subdividido en envejecimiento patológico que es derivado por las secuelas de enfermedades, accidentes o mutilaciones quirúrgicas que cada sujeto ha ido experimentando a lo largo de su vida, o envejecimiento secundario que se relaciona directamente con el tipo de vida que ha llevado el individuo, sus hábitos y sus costumbres (Hoyl et al., 2000).

\section{Cambios relacionados con el envejecimiento}

Se van a producir variaciones en peso y talla, de modo que el peso aumenta entre los 40 y 50 años tendiendo a decrecer a partir de los 70 años. (Kallisont et al., 1997). En cuanto a la talla, ésta disminuye un centímetro por década a partir de la edad adulta. También hay cambios en la composición corporal produciéndose una disminución en la masa magra y un aumento significativo del porcentaje graso y de la masa grasa total. (Mataix y Rivero, 2002; Montero y Ribera, 2002; Ruiz López et al., 2000) al igual que disminuye el agua corporal. La ingesta de energía también disminuye con la edad. A la disminución de la fuerza muscular hay que añadirle los cambios sensoriales como la pérdida de visón y de audición. También hay que añadir los cambios producidos en la capacidad intelectual, como demencias y enfermedad de Alzheimer (Salva et al., 2002).

La reducción del metabolismo de los hidratos de carbono se produce a partir de los 30 años cuando la tolerancia a la glucosa va descendiendo progresivamente (Elahi et al., 2000). En cuanto al metabolismo de los lípidos, existe una acumulación a nivel central y por todo el organismo. La concentración plasmática de colesterol aumenta progresivamente entre los 20-50 años, y posteriormente se estabiliza (Tessari et al., 2001). Respecto al metabolismo proteico, la mayoría de los estudios indican que tanto la síntesis como la degradación de proteínas a nivel corporal no experimentan cambios apreciables en las personas mayores (Tessari, 2001).

Se va a producir también una disminución de la actividad del centro de la sed que puede traducirse en una tendencia a la deshidratación. Así mismo, habrá también cambios morfológicos y fisio-funcionales de los aparatos: engrosamiento de la pared arterial, problemas de tiroides, pérdida de masa ósea, menor producción de saliva, etc.

\section{Valoración del estado nutricional}

Valorar nutricionalmente al anciano nos va a permitir conseguir y mantener un buen estado nutricional en la población anciana, evitando las consecuencias devastadoras de la malnutrición y el desarrollo de patologías asociadas de la obesidad (Valero et al., 2004). Esta valoración es el primer escalón del tratamiento nutricional y 
sus objetivos serán identificar y cuantificar las causas así como las consecuencias de la malnutrición, valorar la morbi-mortalidad que presenta el paciente y valorar si el anciano se beneficiaría de un soporte nutricional (Rexach et al., 2007).

Valoración médica:

Historia clínica (aspectos nutricionales, alteraciones de las funciones sensoriales, patologías, situación funcional del anciano en el momento de la entrevista profundizando en dos aspectos: capacidad de realizar tareas específicas y grado de ayuda necesario para llevarlas a cabo (Rexach et al., 2007).

Determinaciones antropométrica:

Se hace medición del peso, talla, IMC cuyos valores de referencia son: peso insuficiente (IMC $\leq 22)$, normopeso $\left(22-26,9 \mathrm{~kg} / \mathrm{m}^{2}\right)$, sobrepeso $\left(27-29,9 \mathrm{~kg} / \mathrm{m}^{2}\right)$ y obesidad ( $\geq 30 \mathrm{~kg} / \mathrm{m}^{2}$ ), pliegues cutáneos, perímetros, índice de cintura-cadera (ICC) e impedancia bioeléctrica (Polo y Del Castillo).

Parámetros bioquímicos

Se realiza una determinación de las proteínas viscerales: albúmina (3,5-4,8 $\mathrm{mg} / \mathrm{dl})$, prealbúmina y transferrina $(>175 \mathrm{mg} / \mathrm{dl})$ y de las proteínas somáticas: creatinina. También una valoración inmunológica y de hormonas (García et al., 2007; Sergi et al., 2006; Fuhrman et al., 2004).

\section{Valoración de la ingesta dietética}

La valoración de la ingesta dietética se puede realizar mediante dos métodos:

Métodos prospectivos: Pretenden medir la ingesta dietética actual del anciano. Estos son: pesada de alimentos, pesada con análisis químico, registro de consumo (3-7 días), fotográfico e inventario y compra (Torres, 2001).

Métodos retrospectivos: Pretenden medir la ingesta de un pasado inmediato. Estos son: historia dietética, recuerdo de alimentos y frecuencia de alimentos (Planas et al., 2007).

Otros métodos de valoración nutricional utilizados son: el test de Cribado nutricional o Screening nutricional, el Nutrition Screnning Initiative (NSI-Checklist), la Encuesta Nutritional Risk Screnning (NRS), el Mini Nutritional Assessment (MNA), y el test de valoración del estado mental (Mini Mental test de Folstein) (García et al., 2004; Serra et al., 2006; Persson et al., 2002; Guidoz et al., 1994; Arellano et al., 2004; Folstein et al., 1975).

Requerimientos y recomendaciones nutricionales para personas de edad avanzada

Ingestas recomendadas: Las organizaciones internacionales relacionadas con la salud, recomiendan ingerir unas cantidades a las que llaman Ingestas recomendadas (IR) de energía y nutrientes, que aseguran las necesidades del 97,5\% de la población 
sana están cubiertas. Recommended Dietary Allowances. (National Research Council, 1989).

Energía: Las necesidades de energía van disminuyendo con la edad, un 5\% por década (unas 300-600 Kcal). Además se han estimado unas IR de $30 \mathrm{kcal} / \mathrm{kg}$ de peso corporal y podrían situarse en torno a las $2200 \mathrm{kcal} /$ día para los hombres y $1900 \mathrm{kcal} / \mathrm{día}$ para las mujeres (Novartis, 2002).

Macronutrientes:

Proteínas: las IR se establecen en $0,8 \mathrm{~g}$ de proteínas por kg/día. El aporte proteico no debe ser superior al 10-15\% de la energía total consumida aunque en estados hipercatabólicos su aporte puede ser aumentado a un 12-17\%, y disminuido en caso de patologías renales o hepáticas (FAO/OMS, 2003) (Moreiras et al., 2009).

Hidratos de carbono: Se recomienda que aporten el 55-60\% de la energía total consumida (Moreiras et al., 2008). Se deben consumir principalmente hidratos de carbono complejos que están presentes en los cereales, algunas verduras y hortalizas, frutas y las leguminosas.

Fibra dietética: Se recomienda un consumo de 20-30g/día, entre fibra soluble (leguminosas, frutas, algunas verduras, y frutos secos), e insolubles (cereales integrales, pan, verduras...) (Mataix et al., 2002).

Lípidos: El aporte de la grasa total de un 30-35\% de la energía total, pero cuidando la calidad de la grasa ingerida, de forma que los ácidos grasos saturados supongan entre un $7-10 \%$, los ácidos grasos mono insaturados un 15-20\% y los ácidos grasos poliinsaturados $<7 \%$ de las kcal totales. El colesterol no superará los $300 \mathrm{mg} /$ día (Villar et al., 2000).

Micronutrientes: Vitaminas y minerales: En las personas de edad avanzada las necesidades de vitaminas están aumentadas (Carbajal, 2001; Arbonés et al., 2003).

\section{Objetivos}

Se plantea realizar el presente estudio en una residencia de ancianos ubicada en la localidad de Huelma, provincia de Jaén. Los objetivos planteados son los siguientes: Valoración del estado nutricional de la población. Evaluación de la ingesta de la población estudiada de acuerdo a las Ingestas Recomendadas. Valoración del estado antioxidante del menú de la residencia y comparación con la media española.

\section{MATERIAL Y MÉTODO}

El grupo de estudio estuvo constituido por 28 ancianos (8 hombres y 20 mujeres), de edades comprendidas entre 61 y 93 años. El grupo de ancianos estaba institucionalizado en una Residencia para Mayores de carácter concertado. La mayoría de los individuos tenían una capacidad funcional y sensitiva adecuada; de esta manera 
tanto las medidas antropométricas, las encuestas alimentarias y los métodos de evaluación los pudieran contestar los propios individuos.

Se les realizó también una valoración de la dieta durante 2 semanas. Se recogió también, información sobre si tomaban o no suplementos dietéticos, si tenían alergia o intolerancia, si tomaban o no medicación, su ingesta de alcohol o el consumo de tabaco y las enfermedades que padecían y padecieron anteriormente.

Todos los individuos dieron su consentimiento en la participación del estudio de acuerdo con la Declaración de Helsinki (World Medical Association of Helsinki, 1997). Se han tenido en cuenta indicadores socio-demográficos (edad, sexo, estado civil y nivel cultural), datos antropométricos (peso, talla, circunferencias corporales, índice de cintura-cadera, índice de masa corporal, medidas de tensión arterial), cuestionarios de valoración del estado nutricional (MNA, NSI, Test de Pfeiffer, MMSE, índice de Barthel) y valoración de la ingesta para la valoración del estado nutricional de los ancianos (recordatorio de 24 horas y un registro de 7 días).

Se han utilizado los siguientes programas para el análisis de datos: Paquete informático Office. Sistema operativo Windows Vista. Hoja de cálculo Excel. Nutrire 2006 para la valoración de la ingesta semanal de los sujetos, tiene como base de datos las tablas de composición de alimentos de Novartis. Los datos obtenidos se compararon con los de las Ingestas Recomendadas para la población española.

Las variables normales se analizaron por el test de la $t$ de Student para dos muestras. Se han considerado diferencias estadísticamente significativas con $p<0,05$ y en otros casos con $p<0,001$. Los resultados se expresan como media y desviación estándar de la media para el estudio nutricional $(X \pm S D)$. Los análisis se realizaron empleando la hoja de cálculo del Excel 2010.

\section{RESULTADOS Y DISCUSIÓN}

Se ha utilizado el test de la $t$ Student de doble cola para el análisis estadístico, considerándose significativamente diferentes los datos obtenidos con una $p \leq 0.05$ (intervalo de confianza del 95\%).

\section{Parámetros antropométricos}

Tabla 1. Parámetros antropométricos

\begin{tabular}{lcccc}
\hline \multicolumn{1}{c}{ PARÁMETROS ANTROPOMÉTRICOS } & TOTAL \pm DE & HOMBRES \pm DE & MUJERES \pm DE & P. VALOR \\
\hline Edad media población $($ años $)$ & $81,78 \pm 8,28$ & $83,4 \pm 6,11$ & $77,75 \pm 11,13$ & 0,2346 \\
\hline Peso $(\mathrm{kg})$ & $67,83 \pm 4,12$ & $80,37 \pm 6,03$ & $62,82 \pm 13,28$ & $0,0002 * * *$ \\
\hline Talla $(\mathrm{cm})$ & $1,57 \pm 0,095$ & $1,69 \pm 0,072$ & $1,53 \pm 0,062$ & $0,0003 * * *$ \\
\hline IMC $\left(\mathrm{kg} / \mathrm{m}^{2}\right)$ & $27,30 \pm 4,84$ & $28,15 \pm 1,37$ & $26.96 \pm 5,63$ & 0,4103 \\
\hline Perímetro braquial $(\mathrm{cm})$ & $22,32 \pm 3,84$ & $24,12 \pm 3,22$ & $21,6 \pm 3,84$ & 0,1494 \\
\hline Índice cintura/cadera $(\mathrm{cm})$ & $0,91 \pm 0,22$ & $0,94 \pm 0,08$ & $0,90 \pm 0,13$ & 0,3565 \\
\hline TA sistólica & $120 \pm 1,70$ & $130 \pm 1,65$ & $120 \pm 2,56$ & 0,1309 \\
\hline TA diastólica & $70 \pm 9,71$ & $70 \pm 11,11$ & $70 \pm 9,1$ & 0,9584 \\
\hline$* p<0.05 * * * p<0.001$ & & & &
\end{tabular}


GUZMÁN, LÓPEZ y OLIVERAS. Estudio y seguimiento nutricional en una población de ancianos...

Tabla 2. Parámetros antropométricos según rango de edad

\begin{tabular}{lccc}
\hline \multicolumn{1}{c}{ PARÁMETROS ANTROPOMÉTRICOS } & $<65$ AÑOS & $65-75$ AÑOS & $>75$ AÑOS \\
\hline Edad media población $($ años) \pm DE & 61 & $70,5 \pm 2,56$ & $86 \pm 3,85$ \\
\hline Peso $(\mathrm{kg}) \pm \mathrm{DE}$ & 77,7 & $73,33 \pm 17,74$ & $66,41 \pm 12,70$ \\
\hline Talla $(\mathrm{cm}) \pm \mathrm{DE}$ & 1,67 & $1,60 \pm 0,15$ & $1,58 \pm 0,07$ \\
\hline IMC $\left(\mathrm{kg} / \mathrm{m}^{2}\right) \pm \mathrm{DE}$ & 27,8 & $28,25 \pm 5,09$ & $27,19 \pm 4,83$ \\
\hline Perímetro braquial $(\mathrm{cm}) \pm \mathrm{DE}$ & 23 & $22,5 \pm 3,04$ & $21,81 \pm 4,28$ \\
\hline Índice cintura/cadera $(\mathrm{cm}) \pm \mathrm{DE}$ & 0,97 & $0.90 \pm 0,06$ & $0,92 \pm 0,13$ \\
\hline PA sistólica $\pm \mathrm{DE}$ & 110 & $130 \pm 18,63$ & $130 \pm 14,58$ \\
\hline PA diastólica $\pm \mathrm{DE}$ & 60 & $80 \pm 11,05$ & $70 \pm 8,24$ \\
\hline${ }^{*} p<0.05 * * * p<0.001$ & & &
\end{tabular}

Peso: el valor medio del peso de la muestra es de $67,83 \pm 4,12 \mathrm{~kg}$, siendo $80,37 \pm 6,03$ el valor medio de los hombres y $62,82 \pm 13,28$ el de las mujeres. Los hombres presentan un peso significativamente superior al de las mujeres $(p<0,001)$.

Talla: en el colectivo estudiado encontramos que el valor medio de la talla era de $1,57 \pm 0,095 \mathrm{~cm}$. Se ha observado que los varones presentan una talla significativamente superior a la de las mujeres $(p<0,001)$.

IMC: Según sexo, la media de IMC es de $27,30 \pm 4,84 \mathrm{~kg} / \mathrm{m}^{2}$, siendo el valor máximo de $40,6 \mathrm{~kg} / \mathrm{m}^{2}$ y el IMC mínimo de $18,91 \mathrm{~kg} / \mathrm{m}^{2}$, ambos valores en las mujeres. El valor medio de IMC en los varones es superior al de las mujeres. Tabla 1. Por otro lado, el rango de edad dónde mayor IMC hay $\left(28,25 \pm 5,09 \mathrm{~kg} / \mathrm{m}^{2}\right)$ es entre $65-75$ aunque las diferencias no son significativas respecto a los demás grupos de edad. A pesar de que el $32,1 \%$ de la población estudiada esté dentro del criterio de normo peso, hay también un alto porcentaje de personas con sobrepeso y obesidad, $28,6 \%$ y 21,5\% respectivamente, siendo los hombres los que mayor sobrepeso presentan (Tablas 3 y 4 ).

Tabla 3. Distribución de la población por sexo según los valores de IMC

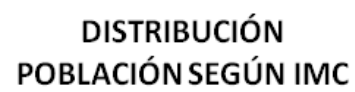

- PESO INSUFICIENTE

- NORMOPESO

SOBREPESO

- OBESIDAD

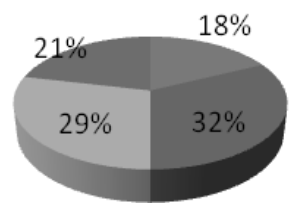

HOMBRES

\section{- PESO INSUFICIENTE}

- NORMOPESO

SOBREPESO

- OBESIDAD

$13 \%$

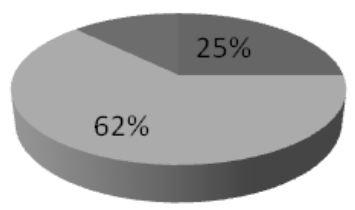

MUJERES

- PESO INSUFICIENTE

- NORMOPESO

- SOBREPESO

- OBESIDAD

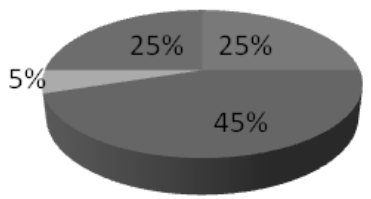


Tabla 4. Distribución de la población por edad según los valores de IMC

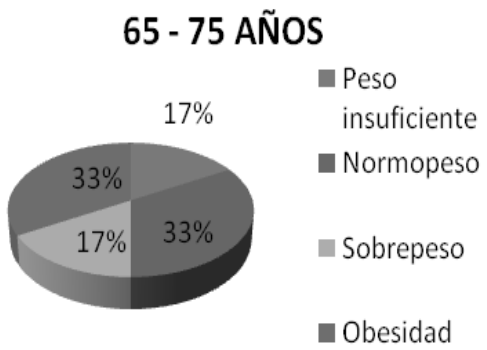

$>75$ AÑOS

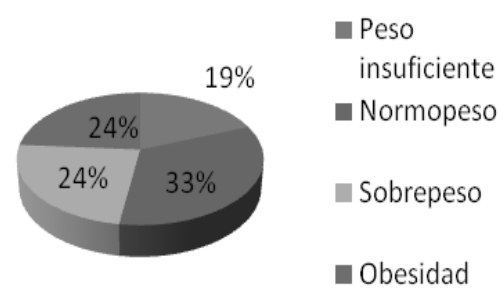

Tabla 5. Comparación de los distintos PB según varios autores

\begin{tabular}{lcc}
\hline \multicolumn{1}{c}{ Perímetro Braquial $(\mathrm{PB})(\mathrm{cm})$} & HOMBRES & MUJERES \\
\hline PRESENTE ESTUDIO & $24,12 \pm 3,22$ & $21,6 \pm 3,84$ \\
\hline GONZÁLEZ HERMOSO & $27,5 \pm 2,6$ & $28 \pm 3,2$ \\
\hline ALASTRUÉ & $24,96 \pm 3,03$ & $25,24 \pm 4,29$ \\
\hline ESQUIUS & 23,87 & 22,12 \\
\hline NUTRICIA & $28,4 \pm 3,7$ & $28,7 \pm 3,8$ \\
\hline JIMÉNEZ-SANZ & $27,1 \pm 2,6$ & $27,1 \pm 4,9$ \\
\hline
\end{tabular}

Índice de cintura-cadera (ICC): Observamos que las mujeres del estudio presentan unos valores de ICC superiores a lo establecido por los valores de referencia según la SEEDO $2008(>0,88 \mathrm{~cm})$ ya que la media es de $0,90 \pm 0,13$, con lo cual el riesgo de padecer enfermedades cardiovasculares está aumentado. En el caso de los varones, la media es de 0,94 $\pm 0,08$ valor por debajo del estándar $(>1 \mathrm{~cm})$; con lo cual no hay riesgo elevado de padecer enfermedades cardiovasculares. No hay diferencias significativas respecto a edades comprendidas entre 65-75 años y los >75 años (Tablas 1 y 2).

Tensión arterial: según los resultados obtenidos, el 13\% de la población masculina tenía hipertensión grado II, sin embargo en las mujeres un $10 \%$ presenta hipertensión grado I. En ambos sexos el $25 \%$ de la población presentó una tensión arterial normalizada.

Perímetro braquial: Se obtuvieron valores medios de 22,32 $\pm 3,84 \mathrm{~cm}$. Para hombres, el valor medio fue de $24,12 \pm 3,22 \mathrm{~cm}$, cifra superior que la de las mujeres con $21,6 \pm 3,84 \mathrm{~cm}$. Tabla 1. Si comparamos estos resultados con los encontrados por otros autores para este tipo de colectivos, observamos que en las mujeres, los resultados obtenidos en el presente estudio se asemejan a los resultados obtenidos por Esquius $(22,12 \mathrm{~cm})$. Sin embargo, respecto a los hombres se asemejan con los obtenidos por Alastrúe $(24,96 \pm 3,03 \mathrm{~cm})$ y Esquius $(23,87 \mathrm{~cm})$ (Tabla 5). 
Estado nutricional, valoración de la ingesta y comparación con las ingestas recomendadas para la población española

Valoración del estado nutricional: La valoración del estado nutricional de los participantes del centro geriátrico se ha calculado a partir de los cuestionarios del estado nutricional MNA y NSI. Por otro lado, para la valoración del estado mental se emplearon los test de Yesavage y MMSE.

MNA: Según los resultados obtenidos a partir del test MNA, se comprobó que el 7,2\% de la población presentaba desnutrición, 50\% riesgo de desnutrición y el 42,8\% estado nutricional normal. El 50\% tanto de varones como de mujeres está en riesgo de desnutrición, y sólo en las mujeres aparece desnutrición siendo este porcentaje del 10\%. Por otro lado el rango de edad donde mayor riesgo de desnutrición hay es en el de $>75$ años. Los datos obtenidos a través del NSI fueron similares al MNA.

MMSE: A partir de los resultados del test MMSE, se obtuvieron los siguientes resultados: el $39 \%$ de la población presentaba deterioro cognitivo, el $22 \%$ estaba bajo sospecha patológica y el $39 \%$ no tenían ningún deterioro cognitivo. No hubo ningún sujeto que presentara demencia. En el grupo de hombres, el 37,5\% tenía deterioro cognitivo, el 12,5\% estuvo bajo sospecha patológica y el 50\% de los ancianos tenía un estado normalizado. Respecto a las mujeres, un $40 \%$ presentó deterioro cognitivo. El grupo de edad con mayor porcentaje de deterioro cognitivo fue el de 65-75 años.

Escala de Yesavage: Se le realizó solo a 17 de las 28 personas ya éstas que fueron las únicas que no presentaron deterioro cognitivo, todas ellas dieron una puntuación dentro del parámetro de normalidad.

Índice de Barthel: Según los resultados obtenidos, el $4 \%$ de la población presentó dependencia grave, el 18\% dependencia moderada, un 58\% dependencia leve y el $21 \%$ de los encuestados presentó total independencia.

\section{Valoración de la ingesta}

Se pretende relacionar la dieta habitual de los ancianos de la residencia (de 2 semanas) con el estado nutricional. Las ingestas se han comparado con las Ingestas Recomendadas para la población española. Los resultados se pueden observar en la tabla 6. La ingesta calórica media de la población es de $2470,15 \pm 82,35 \mathrm{kcal} /$ día. Siendo mayor en hombres $(2552,5 \mathrm{kcal} / \mathrm{día})$ que en mujeres (2387,8 kcal/día). Las ingestas calóricas media tanto en el grupo de los hombres como en el de las mujeres son superiores a las ingestas recomendadas (2200 kcal/día en hombres y $1900 \mathrm{kcal} / \mathrm{d}$ ía en mujeres) (IR según Novartis). Si valoramos la ingesta de proteínas por kg de peso/día, la media fue de $1,32 \mathrm{~g} / \mathrm{kg} /$ día para la población total, ingesta superior a la media española $(0,8 \mathrm{~g} / \mathrm{kg})$. La ingesta de fibra en mujeres es de $23,34 \mathrm{~g} /$ día y la de hombres es de 24,94 $\mathrm{g} /$ día, cifra inferior a la de las recomendaciones. La ingesta de colesterol fue de 403,31 
g/día y 373,37 g/día hombres y mujeres respectivamente, que serían cifras superiores a las recomendaciones (Tabla 6).

Tras evaluar el menú consumido por las personas de la residencia, se observó que el perfil calórico era inadecuado, ya que el porcentaje de grasas consumido tanto en hombres como en mujeres fue ligeramente superior a las recomendaciones; el de carbohidratos, sin embargo fue inferior al recomendado. Por otro lado, el perfil lipídico de la población fue adecuado, manteniendo los porcentajes de AGS, AGMI y AGPI dentro de los valores estándares según las IR. La ingesta de calcio de la población de estudio es de 1040,78 $\pm 32,78 \mathrm{mg} / \mathrm{d}$. Tanto hombres como mujeres que presentan ingestas de $1073 \mathrm{mg} / \mathrm{d}$ y $1008 \mathrm{mg} / \mathrm{d}$ respectivamente, tienen ingestas inferiores a las ingestas adecuadas (IA) de $1200 \mathrm{mg} / \mathrm{d}$ para mujeres y $1300 \mathrm{mg} / \mathrm{d}$ para varones, según la FAO (Cuervo et al., 2009; Novartis, 2006). La ingesta de vitamina A de la población es de $3263,36 \mu \mathrm{g} / \mathrm{d}$, tres veces superior a las ingestas recomendadas $(900 \mu / \mathrm{d}$ en hombres y $700 \mu \mathrm{g} / \mathrm{d}$ en mujeres). La hipervitaminosis está presente en casi todos los tipos de vitaminas excepto en la B9. Además, se han observado diferencias estadísticamente significativas $(p<0,05)$ superiores en los varones respecto a las mujeres en riboflavina. También existen deficiencias en la ingesta de algunas vitaminas $(<2 / 3$ partes $)$ tanto en varones como mujeres, es el caso de las vitaminas $\mathrm{D}$ y $\mathrm{E}$ y ácido fólico con valores de $7,81 \pm 0,22 \mu / \mathrm{d}, 11,27 \pm 0,035 \mu \mathrm{g} / \mathrm{d}$ y $246 \pm 0,035 \mu \mathrm{g} / \mathrm{d}$. respectivamente (Tabla 6).

Tabla 6. Resultados de la ingesta diaria en función del sexo

\begin{tabular}{|c|c|c|c|c|c|c|}
\hline NUTRIENTE & $\begin{array}{l}\text { INGESTA POBLACIÓN } \\
\text { TOTAL } \pm \text { DE }\end{array}$ & $\begin{array}{c}\text { INGESTA } \\
\text { VARONES } \\
\pm \text { DE } \\
\end{array}$ & $\begin{array}{c}\text { INGESTA } \\
\text { MUJERES } \\
\pm \mathrm{DE}\end{array}$ & $\begin{array}{c}\text { IR } \\
\text { HOMBRES }\end{array}$ & $\begin{array}{c}\text { IR } \\
\text { MUJERES }\end{array}$ & P. VALOR \\
\hline Energía (kcal) & $2470,15 \pm 82,35$ & 2552,5 & 2387,8 & 2200 & 1900 & 0,1414 \\
\hline Proteína (g) & $89,77 \pm 4,12$ & 93,9 & 85,68 & 56 & 46 & 0,0192 \\
\hline Lípidos (g) & $98,58 \pm 1,99$ & 100,57 & 96,59 & ND & ND & 0,2560 \\
\hline $\begin{array}{l}\text { Hidratos de } \\
\text { carbono }(\mathrm{g})\end{array}$ & $304,98 \pm 12$ & 316,98 & 292,98 & ND & ND & 0,1207 \\
\hline Fibra $(\mathrm{g})$ & $24,14 \pm 0,80$ & 24,94 & 23,34 & 30 & 21 & 0,1266 \\
\hline Colesterol (g) & $388,34 \pm 14,97$ & 403,31 & 373,37 & 300 & 300 & 0,0890 \\
\hline Calcio (mg) & $1040,78 \pm 32,78$ & 1073,57 & 1008 & 800 & 800 & 0,06587 \\
\hline Hierro (mg) & $15,15 \pm 0,40$ & 15,56 & 14,75 & 8 & 8 & 0,0777 \\
\hline Magnesio (mg) & $273,55 \pm 7,82$ & 281,37 & 265,73 & 320 & 320 & $0,0400^{*}$ \\
\hline Zinc (mg) & $9,59 \pm 0,57$ & 10,17 & 9,02 & 9 & 9 & 0,0889 \\
\hline Fósforo (mg) & $1420,49 \pm 45,31$ & 1465,81 & 1375,18 & 700 & 700 & 0,6323 \\
\hline Tiamina (mg) & $2,46 \pm 0,035$ & 2,5 & 2,43 & 1,2 & 1,1 & 0,0713 \\
\hline $\begin{array}{l}\text { Riboflavina } \\
(\mathrm{mg})\end{array}$ & $1,89 \pm 0,055$ & 1,95 & 1,84 & 1,3 & 1,1 & 0,0889 \\
\hline Niacina $(\mathrm{mg})$ & $19,44 \pm 0,63$ & 20,08 & 18,81 & 16 & 14 & $0,0468 *$ \\
\hline Vitamina A $(\mu \mathrm{g})$ & $3159,64 \pm 103,71$ & 3263,36 & 3055,93 & 900 & 700 & 0,0656 \\
\hline Vitamina $C(\mu \mathrm{g})$ & $212,28 \pm 2,38$ & 214,66 & 209,9 & 90 & 75 & 0,0898 \\
\hline Vitamina D $(\mu \mathrm{g})$ & $7,81 \pm 0,22$ & 8,03 & 7,59 & $15^{*}$ & $15^{*}$ & 0,0767 \\
\hline Vitamina E $(\mu \mathrm{g})$ & $11.27 \pm 0,035$ & 11,32 & 11,24 & 15 & 15 & 0,0744 \\
\hline $\begin{array}{l}\text { Piridoxina B6 } \\
(\mathrm{mg})\end{array}$ & $1,93 \pm 0,01$ & 1,94 & 1,92 & 1,7 & 1,5 & 0,1112 \\
\hline $\begin{array}{l}\text { Cianocobalamin } \\
\text { a B12 }(\mu \mathrm{g})\end{array}$ & $6,31 \pm 0,40$ & 6,72 & 5,91 & 2,4 & 2,4 & $0,0484 *$ \\
\hline $\begin{array}{l}\text { Ácido Fólico B9 } \\
(\mu \mathrm{g})\end{array}$ & $246,26 \pm 686$ & 253,13 & 239,4 & 400 & 400 & 0,0611 \\
\hline
\end{tabular}




\section{Capacidad antioxidante de la dieta}

La CAT de las mujeres es de 30611 CAT/día y la de los varones de 32143 CAT/día. La media de CAT de los españoles utilizando valores de capacidad antioxidante lipo-hidrofílica (Wu et al., 2005) disponibles según la técnica ORAC (oxigen radical absorbance capacity) y expresados en micromoles equivalente de Trolox por gramo es de 10.577. Respecto al contenido de polifenoles, la dieta de los ancianos muestra unos valores de $2344 \mathrm{mg} /$ día, valor que duplica el valor medio de la población española de 1171 mg/día (Saura-Calixto y Goñi, 2006).

\section{CONCLUSIONES}

Las conclusiones obtenidas a partir de los resultados anteriores son las siguientes:

Peso: La mayoría de los ancianos tienen normo peso y sobrepeso con un de $32 \%$ y $29 \%$ respectivamente. Hay un mayor porcentaje de hombres con sobrepeso que de mujeres, sin embargo las mujeres son las únicas que tienen peso insuficiente. Índice de cintura cadera (ICC): Del estudio se deduce que las mujeres tienen aumentado el riesgo de padecer enfermedades cardiovasculares, a diferencia de los varones. Tensión arterial: La tensión arterial es normal, con la excepción de hipertensión grado I observada en 2 casos y la hipertensión grado II observada en un anciano.

Según MNA, el 50\% de la población presenta riesgo de desnutrición siendo este porcentaje significativo y equiparado tanto en mujeres como en hombres. El grupo de población de $>75$ años es el que más riesgo presenta.

En casi la mitad de los ancianos institucionalizados se observa sospecha patológica de deterioro cognitivo. Sin embargo no hay diferencias estadísticamente significativas entre hombres y mujeres.

Más de la mitad de los ancianos presentan dependencia leve, efecto mayor en las mujeres y rango de edad de $>75$ años.

Ingesta calórica: la ingesta calórica de esta población supera la ingesta recomendada en un $15 \%$ y un $30 \%$ hombres y mujeres respectivamente. El perfil calórico es inadecuado, con exceso de grasas e ingesta baja en carbohidratos. Las proteínas se mantienen en niveles adecuados. El perfil lipídico es adecuado. La ingesta de fibra en hombres en inferior a las recomendaciones, en mujeres es adecuada. Hay deficiencias en micronutrientes como el yodo, magnesio, vitamina D, E y ácido fólico. Estas deficiencias son ligeramente superiores en mujeres que en hombres. El colesterol está elevado tanto en mujeres como en hombres.

La capacidad antioxidante de la dieta de los ancianos triplica los valores medios establecidos para la población, por otra parte, el contenido de polifenoles es dos veces superior a la media establecida para la población española. 


\section{REFERENCIAS}

Abajo, C., García, S., Calabozo, B., Ausin, L., Casado, J. y Catalá, M.A. (2008). Protocolo de valoración, seguimiento nutricional en un centro residencia para personas mayores. Nutrición Hospitalaria, 23(2), 100-104.

Álvarez, J.R. y Pulido, M. (2010). Capacidad antioxidante de la dieta española. La rueda de los alimentos antioxidantes. Rev. Esp. Sociedad Española de Dietética y Ciencia de la Alimentación, 1, 9-15.

Aranceta, J., Serra-Majem, L., Arija. V., Gil, A., Martínez de Vitoria, E. y Ortega, R. (2011). Objetivos Nutricionales para la población española. Rev. Esp. Nutr. Comunitaria, 17(4) 178-199.

Bellido, D. y De Luis, D.A. (2000). Manual sobre el Consenso (SEEDO 2000) para la evaluación del sobrepeso y la obesidad y el establecimiento de criterios de intervención terapéutica. Medicina Clínica, 115, 587-597.

Caballero, J.C., Benítez, J., Aranceta, J., Artaza, I., Astudillo, W., Barrallo, T., Castro, J. y García P. (2011). Manual de atención al anciano desnutrido en el nivel primario de salud. Medicina Clínica, 211, 344-356.

Castillo, C, Uauy, R. y Atalh, E. (1999). Manual de evaluación del estado nutricional en el anciano. Guías de alimentación para el adulto mayor. Medicina Clínica, 567, 39-52.

Esquius, M., Schwartz, S., López, J. y Andréu, A.L. (1993). Parámetros antropométricos de referencia de la población anciana. Medicina Clínica, 100(18), 692-698.

García de Lorenzo, A. y Ruipérez, I. Valoración nutricional en el anciano. Recomendaciones prácticas de los expertos en geriatría y nutrición. SENPE (Sociedad Española de Nutrición Parenteral y Enteral), SEGG (Sociedad Española de Geriatría y Gerontología).

García P. y Serra, J. (2007). Manual de recomendaciones nutricionales en pacientes geriátricos. Nutrición Hospitalaria, 23(2), 43-49.

Martín, C. (2001). Ingestas Recomendadas en personas de edad avanzada. Alimentación, Nutrición y Salud, 8(4), 100-114.

Serra, L. y Aranceta J. (2008). Objetivos nutricionales para la población española. Consenso de la Sociedad Española de Nutrición Comunitaria. Madrid: Mapya.

Valero, M.A. y León, M. (2004). Recomendaciones nutricionales en pacientes geriátricos. Zaragoza: Acribia.

Recibido: 9 de abril de 2014 Recepción Modificaciones: 26 de mayo de 2014

Aceptado: 1 de junio de 2014 